\title{
Altered static and dynamic functional connectivity in subthreshold bulimia nervosa: A resting-state functional magnetic resonance imaging(fMRI) study
}

Lin Luo

Southwest University

Yijun Luo

Southwest University

Ximei Chen

Southwest University

Mingyue Xiao

Southwest University

Ziming Bian

Southwest University

Xuechen Leng

Southwest University

Wei Li

Southwest University

Junjie Wang

Southwest University

Yue Yang

Southwest University

Hong Chen ( $\square$ chenhswu@163.com )

Southwest University https://orcid.org/0000-0001-8888-5103

\section{Research Article}

Keywords: bulimia nervosa, degree centrality, static functional connectivity, dynamic functional connectivity, resting state functional magnetic resonance imaging

Posted Date: August 24th, 2021

DOI: https://doi.org/10.21203/rs.3.rs-823131/v1

License: () (i) This work is licensed under a Creative Commons Attribution 4.0 International License.

Read Full License 


\section{Abstract}

Bulimia nervosa (BN) is one of the most serious eating disorders (EDs) associated with long-term significant impairment to both physical and mental quality of life. Subthreshold BN emphasize that individuals reported some extent bulimic symptoms, even those individuals who did not fully meet the diagnostic criteria endure elevated levels of impulsivity and higher likelihood of self-harm. Therefore, the current study aimed to identify potential neurobiological signatures of high-risk individuals with BN symptoms. We used functional magnetic resonance imaging ( $\mathrm{fMRI}$ ) to investigate the differences between a subthreshold BN group (sub-BN, $n=61$ ) and a control group $(n=51)$ in terms of degree centrality (DC), with the secondary analysis of the connectivity in these ROls showing differences in both static functional connectivity (sFC) and dynamic functional connectivity (dFC) with age, sex, and framewise displacement (FD) as covariates of no interest ( $p<0.05$, GRF corrected). The sub-BN group exhibited significantly increased DC in the left middle frontal gyrus (MFG) and the orbital frontal gyrus, increased MFG-superior occipital gyrus connectivity, MFG-middle occipital gyrus connectivity and MFG-triangular part of the inferior frontal gyrus (IFGtriang) connectivity, and decreased variability in MFG-anterior cingulate cortex connectivity. Furthermore, in the sub-BN group, a positive correlation was observed between self-reported BN symptoms and MFG-IFGtriang connectivity. These findings implicated in reward value processing, cognitive control, and self-referential processing in individuals with BN symptoms, which provided important insights into understanding the pathology of BN.

\section{1 | Introduction}

Bulimia nervosa (BN) is characterised by compensatory behaviours intended to avoid weight gain after recurrent episodes of binge eating; these include self-induced vomiting, laxative and/or diuretics abuse, fasting, and excessive physical exercise (Mele, Alfano, Cotugno, \& Longarzo, 2020). The lifetime prevalence rates of $\mathrm{BN}$ range from $4-6.7 \%$, although there has been an increase in disordered eating that does not necessarily meet the BN diagnostic criteria (Wade, 2019). Previous studies have mostly investigated the neurobiological underpinnings of $\mathrm{BN}$ in clinical conditions. Less is known about the spontaneous neural basis involved in subthreshold BN (sub-BN). Given that the presence of disordered eating confers a risk of elevated levels of impulsivity and a higher likelihood of self-harm (Wade, 2019), there is a need to investigate the neurological substrates of sub-BN in order to understand the neural basis of $\mathrm{BN}$, and to contribute to the prevention of and intervention for $\mathrm{BN}$.

In terms of neurobiology, BN commonly exhibits abnormalities in the reward and cognitive control systems. In the reward system, patients with BN show increased activity in the medial orbitofrontal cortex (OFC) in response to food images (Brooks et al., 2011) and during food reward (Simon et al., 2016), which is one of the most important brain regions for food reward processing (Burger \& Stice, 2011). Abnormal activity of these brain circuits may represent higher impulsivity and hedonic processing during receipt of food reward, at least indicating a possibly altered reward sensitivity in BN patients. Additionally, in related to cognitive control system, evidence has shown a relationship between eating restraint scores and heightened activation in the dorsolateral prefrontal cortex (dIPFC), anterior cingulate cortex (ACC), and 
OFC in response to high-calorie food (Wood et al., 2016). Theoretically, individuals with BN symptoms, who are characterised by compensatory behaviours to avoid weight gain, have the same motivation for weight loss as individuals showing eating restraint. Moreover, empirical research has indicated that individuals with BN show greater neural activation in the right dIPFC in response to food images than do HCs (Brooks et al., 2011). Another study reported that individuals with BN demonstrated hyperactivation in the middle frontal gyrus (MFG) within the executive network, as compared to those with binge eating disorder (BED) (Stopyra et al., 2019). Increased activity of the MFG may imply altered cognitive control resources in $\mathrm{BN}$ and reflects a possible means of modulating food-related decisions.

Furthermore, contemporary research has emphasised the importance of connections between spatially distributed brain areas. Indeed, a number of functional connectivity (FC) alterations have been found in patients with BN. Compared to HCs, stronger synchronous activity between the dorsal ACC (dACC) and medial OFC has been demonstrated in individuals with BN through resting-state functional magnetic resonance imaging (fMRl; Lee et al., 2014). Wang et al. (2019) also found increased left-lateralized connections primarily within the orbitofrontal cortex and its connections to mesocorticolimbic and lateral temporal-occipital areas. Another study observed increased FC between the right ventral supramarginal gyrus and all default mode network (DMN) regions in patients with BN (Domakonda, He, Lee, Cyr, \& Marsh, 2019). The $D M N$ is responsible for a self-referential introspective state (Mak et al., 2017) and typically associated with goal-oriented tasks that require self-referential processing (Vatansever et al., 2018), which is involved in regulating physical appearance (Davey, Pujol, \& Harrison, 2016), suggesting that patients with BN may show excessive self-introspection, contributing to the preoccupation with body image (Benninghoven, Raykowski, Solzbacher, Kunzendorf, \& Jantschek, 2007). Moreover, according to the the goal conflict model of eating, which posits that restrained eaters is characterized by a conflict between two chronically accessible incentives or goals: eating enjoyment and weight control (Stroebe, Mensink, Aarts, Schut, \& Kruglanski, 2008). Similarly, individuals with BN symptoms may experience more conflict between hedonic processing of food and self-regulation processing derived from self-referential processing of body weight/shape than do HCs.

The proportion of individuals with sub-BN is larger than clinically diagnosed patients with $\mathrm{BN}$, and individuals with sub-BN are at an increased risk of developing bulimia (Bernadett et al., 2016; Wade, 2019). Previous studies have mainly focused on patients with clinical BN and the aberrant neural correlates of this condition (Simon et al., 2016; Domakonda et al., 2019; Stopyra et al., 2019), ignoring the potential neurobiological signatures in those individuals with high risk of developing clinical BN. Nevertheless, elucidating the neural basis of sub-BN may contribute to identifying risk factors specific to BN for advancing etiological knowledge and designing effective prevention programs. To the best of our knowledge, BN tends to emerge around the age of 19 years (Wade, 2019), and the incidence of BN in the Chinese population has shown an increasing trend, mostly in adolescents (Duan, Xu, \& Li, 2020). Thus, the young adults is a high-risk group for BN in modern times. Therefore, in this study, we selected college students who are at increased risk of $\mathrm{BN}$ to investigate potential brain function changes. Additionally, from the perspective of cognitive behavioural models of $B N, B N$ is characterised by low self-esteem that is linked with individuals' high levels of body weight and shape concerns, thereby contributing to the 
development and maintenance of BN (Meijboom, Jansen, Kampman, \& Schouten, 1999). Therefore, we measured individuals' with sub-BN body esteem and indicators related to weight concerns for further distinction from control group.

Degree centrality (DC) measures the number of direct connections between a given node and the rest of the brain within the entire connectivity matrix of the brain. Although static FC (sFC), which measures the synchronisation of different regional spontaneous neuronal signals and reflects the tendency of cortical networks to be co-activated (Mennes et al., 2018; Worsley \& Friston, 1995), has been widely used in previous studies, it may not be sufficient to fully characterise human brain activity. Human brain connectivity is dynamic and is associated with ongoing rhythmic activity, rather than being static (Calhoun, Miller, Pearlson, \& Adali, 2014; Preti, Bolton, \&Van de Ville, 2017). An emerging method to assess this is dynamic FC (dFC) (Hutchison et al., 2013), which can be investigated by measuring variability in the strength or spatial dynamic organisation of FC.

The present study focused on individuals at high risk of BN and sought to understand potential brain mechanisms that could contribute to preventing or decreasing the prevalence rates of clinical BN. Specifically, we examined changes in brain activity in college-aged individuals with sub-BN by using DC, then with the secondary analysis of the connectivity in these ROls showing differences in both $\mathrm{SFC}$ and $\mathrm{dFC}$. Furthermore, we investigated the potential relationship between FC alterations and self-reported BN symptoms (i.e. BN scores).

\section{2 | Materials And Methods 2.1 | Participants}

All data of current study was part of Behavioural Brain Research Project of Chinese Personality conducted at Southwest University Brain Imaging Center, Chongqing, China. All experimental operations were approved by the Southwest University Brain Imaging Center's Institutional Review Board and all participants provided written informed consent. A total of 646 undergraduates completed self-report questionnaire investigating eating disorder symptoms (Eating Disorder Diagnostic Scale, EDDS, Stice, Telch, \& Rizvi, 2000) and fMRI scanning ,we finally screened out 61 participants (sub-BN group; mean age $=19$ years; 50 females ) with BN symptoms and 51 participants (control group; mean age $=19$ years; 23 females), from the same setting (Chongqing, China), who failed to meet criteria for any eating disorder (ED; that is, anorexia nervosa [AN], BN, or BED) on a widely used EDDS screen. All participants were righthanded, with normal or corrected visual acuity, and without a history of drug or alcohol abuse. No participants were excluded because their framewise displacement (FD) was less than $3 \mathrm{~mm}$ in any direction. The EDDS (Stice, Telch, \& Rizvi, 2000) is a 22-item self-report screening tool based on the Diagnostic and Statistical Manual of Mental Disorders (DSM). We used the sum of $z$-scores for EDDS items related to $\mathrm{BN}$ diagnosis (items $3,4,5,6,8,15,16,17$, and 18) to represent the severity of BN symptoms. All sub-BN group individuals reported at least one compensatory behaviour per week following binge-eating over the previous three months (Lyu, Zheng, \& Jackson, 2016); none of these individuals fully 
met clinical diagnostic criteria for BN. Conversely, none of the control group members reported weekly binge-eating episodes over the past 3 months, or met any EDDS items reflecting a DSM-diagnosis of any ED.

\section{2 | Behavioural measures}

Due to our diagnosis of the symptoms group is self-reported and preliminary, the current study employ some related behavioral performance to validate and strengthen the differences between sub-BN group and control group.

\subsection{1 | EDDS}

The EDDS self-reporting scale consists of 22 items and is based on DSM criteria for AN, BN, and BED. EDDS was used in this study to identify sub-BN participants and to rule out an ED diagnosis among those in the control group. This scale has excellent reliability, a high level of stability over 2 weeks, and excellent concordance with diagnoses based on structured interviews and self-report measures of disordered eating (Stice, Fisher, \& Martinez, 2004; Stice et al., 2000). A back-translated version of the EDDS has been reported to be valid and reliable in epidemiological and longitudinal studies of mainland Chinese adolescents and young adults (Chen \& Jackson, 2008). An overall symptom composite calculated from the sum of $z$-scores for the first 18 EDDS items also provided a severity rating. The 18 -item EDDS composite had internal consistency $a=0.84$. We used nine items (items $3,4,5,6,8,15,16,17$, and 18) of the scale to assess the severity of BN.

\subsection{2 | Negative Physical Self Scale}

The Negative Physical Self Scale (NPSS; Chen, Jackson \& Huang, 2006) was used to assess attitude towards the physical self, using five different dimensions. In this study, we used one dimension for overall appearance as an indicator to measure individuals' satisfaction with their own body (for example, 'I am proud of my body'; 'I have no regrets about my body'). Items were rated from ' 1 = completely incompatible' to ' 5 = completely compatible'; this 5 -item dimension had an $a=0.83$.

\subsection{3 | Body-Esteem Scale}

The Body-Esteem Scale (Mendelson \& White, 1996) consists of eight items; a sample item is 'I think I have a good body'. Responses were scored on a 5-point Likert-type scale ranging from 0 (never) to 4 (always). All items were averaged to a single score, with higher mean scores indicating higher body esteem. In this study, the scale had an $a=0.83$.

\subsection{4 | Restraint Scale}

The Restraint Scale (Kong et al., 2013) is comprised of two subscales. The items in the first subscale pertain to the respondent's dietary concerns and had an internal consistency coefficient of $a=0.67$. The items in the second subscale are related to the participant's weight changes and had an internal consistency coefficient of $a=0.75$. The correlation between the scores on the two subscales was $r=0.40$, indicating an adequate internal reliability. This scale reflected the degree of eating restraint and identified 
individuals who were chronically concerned with their weight and restricted food intake as a means of weight control.

\section{3 | Resting state functional magnetic resonance imaging data acquisition and pre-processing}

\subsection{1 | Resting state functional magnetic resonance imaging data acquisition}

Functional magnetic resonance imaging (fMRI) data were collected during a total of 8 minutes in the resting state on a 3-T Trio scanner (SIEMENS PRISMA, Erlangen, Germany). Functional images were acquired by using a gradient echo planar imaging sequence (repetition time [TR] = 2000 ms; echo time [TE] = $30 \mathrm{~ms}$; slices $=62$; slice thickness $=2 \mathrm{~mm}$; field of view read $=224 \times 224 \mathrm{~mm}^{2} ;$ flip angle $=90^{\circ}$; resolution matrix = $112 \times 112$; voxel size $=2 \times 2 \times 2 \mathrm{~mm}^{3}$; phase-encoding direction $=\mathrm{PC}>>$ AC; volumes $=240$ ) . Additionally, structural images were acquired by using a 3-dimensional magnetisation-prepared rapid gradient-echo (sequence $\left(T R=2530 \mathrm{~ms} ; \mathrm{TE}=2.98 \mathrm{~ms}\right.$; field-of-view $=256 \times 256 \mathrm{~mm}^{2}$; flip angle $=7^{\circ}$; base resolution $=256 \times 256$; slices per slab = 192; slice oversampling $=33.3 \%$; voxel size $=0.5 \times 0.5 \times 1 \mathrm{~mm}^{3}$; phase-encoding direction $=A C>>P C$; volumes $=240$ ). During the resting-state scanning, foam pads and earplugs were employed to reduce head motion and scanning noise. Participants were instructed to maintain an awakened state with their eyes closed and to not think about anything in particular.

\subsection{2 | fMRI pre-processing}

Data were pre-processed using the Data Processing \& Analysis for (Resting-State) Brain Imaging (DPABl; Yan et al., 2016), which employed statistical parametric mapping software (SPM12, http://www.fil.ion.ucl.ac.uk/spm). Images from the first 10 volumes at the beginning of the resting-state scanning were discarded to ensure a steady-state longitudinal magnetisation. Subsequently, we applied slice-timing and motion-correction for the remaining 230 frames. Slice-timing and head motion -correction were performed to correct slice order and head motion effects, respectively. Then, by using the EPI templates in SPM12 (Ashburner, 2007), each image volume was spatially normalised to the Montreal Neurological Institute (MNI)-152 brain template. The normalised images were re-sliced at a resolution of 3 $\times 3 \times 3 \mathrm{~mm}^{3}$ and spatially smoothed with a 4-mm full-width at half-maximum. Next, nuisance signals (that is, white matter, cerebrospinal fluid, and head motion parameters) were regressed out using multiple linear regression analysis. Their derivatives were also regressed using a Friston 24-parameter model to control the effects of potential physiological artifacts (Friston, Williams, Howard, Frackowiak, \&Turner, 1996; Hallquist, Whang, \& Luna, 2013). Finally, data were temporal band-pass filtered $(0.01-0.08 \mathrm{~Hz})$ to reduce the effects of low-frequency drift and physiological high-frequency noise. Furthermore, we implemented data scrubbing to address head motion concerns more effectively. Bad time points were regarded as regressors that were defined as volumes with FD. FD $>0.5 \mathrm{~mm}$, as well as the two succeeding volumes and one preceding volume, were omitted to reduce the spill-over effect of head motion (Power, Barnes, Snyder, Schlaggar, \& Petersen, 2012). 
For all participants, there were no scans with head motion between volumes in any direction $>3 \mathrm{~mm}$ or rotation > 3 degrees in any axis during scanning. The mean FD values did not exceed 0.50; hence no participants were excluded during pre-processing.

\section{4 | Between-group analysis in DC}

As one of the graph-based measurements of network organization, global brain connectivity through DC measures the number of direct connections between a given node and the rest of the brain, within the entire connectivity matrix of the brain. DC was calculated based on previously described methods (Zhou et al., 2014). Briefly, Pearson's correlation coefficients ( $r$ ) were computed for each of the time courses of each pair of voxels in the whole brain. Each voxel was considered as one node in an $\mathrm{n} \times \mathrm{n}$ connection matrix with a connection threshold of $r>0.25$. The binarised DC values were computed for each voxel as the summed number of suprathreshold correlations. Finally, the $z$ transformation was then implemented to improve normality. A two-sample t-test was used to investigate differences in DC between sub-BN and HC individuals, with age, sex, and FD as covariates of no interest. Multiple comparison correction was implemented with a Gaussian random field (GRF) corrected at cluster level $p<0.05$ and voxel level $p<$ 0.005 as the statistical threshold (two-tailed).

\section{5 | Between-group analysis of sFC}

sFC was analysed via a seed-based whole-brain approach using the DPABI software. The significant brain regions from the between-group analysis of DC were chosen as the seed regions (that is, 6-mm-radius spheres in MNI space). We first calculated the linear correlations between the mean time series of each seed region and the time series of all other voxels in the brain. Fisher's $z$ transformation was then applied to the individual seed connectivity maps (Fox \& Raichle, 2007). The obtained $z$ maps were then entered into multiple linear regression analyses to identify the brain regions with a resting state $\mathrm{FC}$ within a given seed region. To identify the sFC differences between two groups, we used a two-sample $t$-test with age, sex, and FD as covariates of no interest. Multiple comparison correction was implemented with a GRF corrected at cluster level $p<0.05$ and voxel level $p<0.005$ as the statistical threshold (two-tailed).

Subsequently, the potential relationships between brain regions of sFC and BN-severity scores in the subBN group were assessed, with age, sex, body mass index (BMI), and FD as covariates. The statistical significance level for the correlation analysis was set at $p<0.05$.

\section{6 | Between-group analysis of dFC variability}

dFC was also analysed via a seed-based whole-brain approach using the DPABI software. The significant brain regions from the between-group analysis of DC were chosen as the seed regions (that is, 6-mmradius spheres in $\mathrm{MNI}$ space). To investigate the $\mathrm{dFC}$ variability of the seeds to the whole brain, a sliding window dFC approach was used in the DynamicBC toolbox (Liao et al., 2014). In the sliding window-based $\mathrm{dFC}$ analysis, window length is an open area of research and is an important parameter. It is worth noting that the minimum window length should be no less than $1 /$ minimum frequency (fmin), because short time segments may introduce spurious fluctuations. In this relation, fmin was the minimum frequency of the correlating time courses (Leonardi \& Van De Ville, 2015). In this study, we examined sliding window length 
of 30 TR (shifting step size was 1 TR) to optimise the balance between capturing a rapidly shifting dynamic relationship (with shorter windows) and achieving reliable estimates of the correlations between regions (with longer windows). The variance of the time series of the correlation coefficient was computed by calculating the standard deviation of $z$ values at each voxel to assess the dFC variability. To assess the $\mathrm{dFC}$ differences between groups, we used a two-sample $t$-test with age, sex, and FD as covariates of no interest. Multiple comparison correction was implemented with a GRF-corrected at cluster level $p<0.05$ and voxel level $p<0.005$ as the statistical threshold (two-tailed).

\section{7 | Correlational analysis}

To assess the potential relationship between the self-reported BN symptoms and altered regions connected with regions of interest, we extracted eigenvariates from the resultant regions (that is, $\mathrm{SFC}$ and $\mathrm{dFC}$ ) from between-group analyses. Partial correlation coefficients were calculated to test for the potential association between BN-severity scores and altered FC, with age, sex, and FD as covariates of no interest.

\section{3 | Results}

\section{1 | Demographics and neuropsychological results}

Demographics of the participants are shown in Table 1. The sub-BN group consisted of 48 women and 13 men, while there were 23 women and 28 men in the control group. No significant between-group differences in age and head motion were found and the average BMI in two groups were within the normal range. In addition, supporting the distinctiveness of the selected groups based on initial EDDS responses, sub-BN group had significantly lower body esteem and overall satisfaction with their appearance than did the control group $(p<0.001$; Table 1$)$. However, the sub-BN group exhibited higher dietary concerns and weight fluctuation scores than the control group $(p<0.001$; Table 1$)$. 
Table 1

Demographic characteristics of the participants

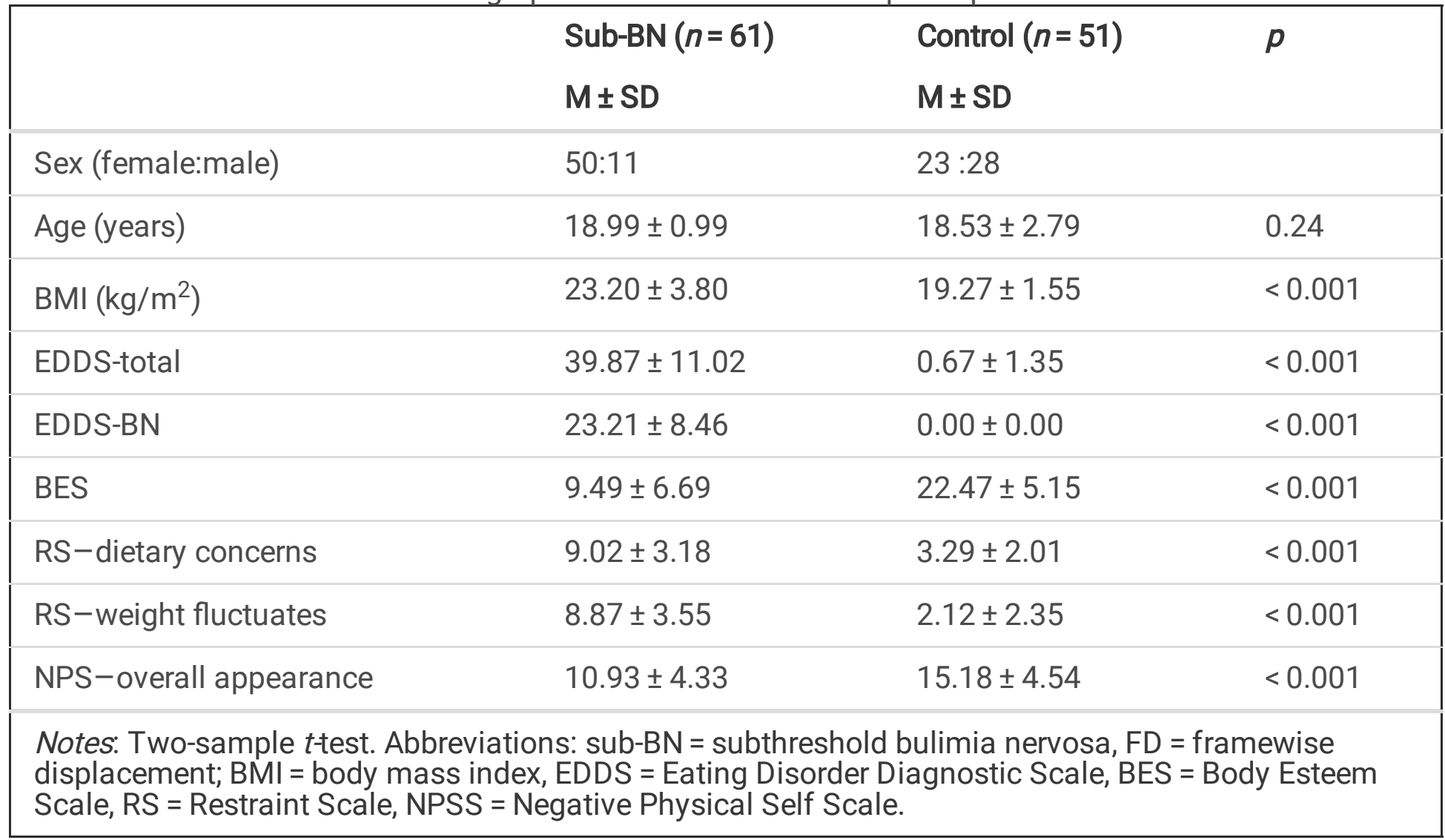

\section{2 | Between-group differences in DC}

The two -sample $t$-test showed that, compared with the control group, the sub-BN group displayed greater DC in the left MFG $(-36,54,0)$ and the left middle orbital frontal gyrus (OFG; $-45,45,-9)$, GRF-corrected at cluster level $p<0.05$ and voxel level $p<0.005$, two-tailed (see Fig. 1 and Table 2).

Table 2

Between-group differences in DC

\begin{tabular}{|lcccccccc|}
\hline Groups/Brain regions & H & BA & $\mathbf{x}$ & y & $\mathbf{z}$ & voxel size & T-value \\
\hline Sub-BN $>$ Control & & & & & & & 3.86 \\
\hline Middle frontal gyrus & L & 10 & -36 & 54 & 0 & 10 & 4.08 \\
\hline Middle frontal gyrus, orbital & L & 11 & -45 & 45 & -9 & 9 & \\
\hline $\begin{array}{l}\text { Notes: Two-sample } t \text {-test. Abbreviations: DC = degree centrality, sub-BN = subthreshold bulimia } \\
\text { nervosa, } H=\text { hemisphere, } L=\text { left, BA = Brodmann area, GRF correction } p<0.05 \text { at cluster level and } p< \\
0.005 \text { at voxel level, 2-tailed. }\end{array}$
\end{tabular}

\section{3 | Between-group differences in sFC}

With the MFG and the OFG being chosen as the seed regions (that is, 6-mm-radius spheres), the sub-BN group displayed significantly increased connectivity between the MFG and the superior occipital gyrus (SOG), middle occipital gyrus (MOG), and triangular part of the inferior frontal gyrus (IFGtriang), as 
compared to the HC group. The results are shown in Fig. 2(a) and Table 3. Finally, the correlational analyses in the sub-BN group revealed a positive correlation between the MFG-IFGtriang connectivity and BN scores $(r=0.30, p<0.05)$ [Fig. 2(b)]. No significant correlation was found between the self-reported BN scores and MFG-SOG connectivity or MFG-MOG connectivity.

Table 3

Functional connectivity differences in the MFG between groups

\begin{tabular}{|c|c|c|c|c|c|c|c|}
\hline Groups/Brain regions & $\mathrm{H}$ & $\mathrm{BA}$ & $x$ & $\mathrm{Y}$ & $\mathbf{Z}$ & voxel size & $T$-value \\
\hline \multicolumn{8}{|l|}{ Sub-BN > Control } \\
\hline \multicolumn{8}{|l|}{ sFC } \\
\hline Middle occipital gyrus & $\mathrm{L}$ & 37 & -45 & -72 & 15 & 110 & 4.15 \\
\hline Superior occipital gyrus & $\mathrm{L}$ & 19 & -24 & -69 & 33 & 90 & 4.36 \\
\hline triangular part of the inferior frontal gyrus & $\mathrm{L}$ & 48 & -42 & 21 & 27 & 58 & 4.33 \\
\hline \multicolumn{8}{|l|}{$\mathrm{dFC}$} \\
\hline Anterior cingulate cortex & $\mathrm{R}$ & 24 & 3 & 30 & 27 & 14 & -3.864 \\
\hline \multicolumn{8}{|c|}{$\begin{array}{l}\text { Notes: Two-sample } t \text { test. Abbreviations: } \mathrm{MFG}=\text { middle frontal gyrus, sub-BN }=\text { subthreshold bulimia } \\
\text { nervosa, } \mathrm{H}=\text { hemisphere, } \mathrm{L}=\text { left; } \mathrm{BA}=\mathrm{Brodmann} \text { area, } \mathrm{SFC}=\text { static functional connectivity, } \mathrm{dFC}= \\
\text { dynamic functional connectivity, GRF correction } p<0.05 \text { at cluster level and } p<0.005 \text { at voxel level, } 2- \\
\text { tailed. }\end{array}$} \\
\hline
\end{tabular}

\section{4 | Between-group differences in dFC variability}

Consistent with the $\mathrm{SFC}$, no significant dFC between-group differences were found between the OFG and the rest of the brain. For the MFG seed, the sub-BN group exhibited decreased dFC variability in the right ACC (GRF-corrected at cluster level $p<0.05$ and voxel level $p<0.005$, two-tailed). The results are presented in Fig. 3 and Table 3. Finally, the correlational analyses in the sub-BN group revealed no significant correlation between self-reported BN scores and the MFG-ACC connectivity.

\section{4 | Discussion}

The present study used resting-state fMRI to examine altered brain bold activity and connectivity in individuals with BN symptoms. We observed group differences in DC, with the secondary analysis of the connectivity in one ROI showing differences in both SFC and dFC. Notably, there was significantly increased activation in the reward and cognitive control areas -OFG and MFG respectively- and in the connections of these areas with self-referential and conflict monitoring processing. Our findings suggested that individuals with sub-BN exhibited hyperactivation of the brain's reward and control systems, to some extent supporting the goal conflict model of eating in BN. Furthermore, aberrant MFG-IFGtriang connectivity in the sub-BN group was associated with their self-reported BN symptoms. 
On the one hand, We found significantly increased DC in the reward system area (OFG). The orbitofrontal lobe is among the most important brain regions for food reward processing (Burger \& Stice, 2011), and is related to the expectation of reward value and value-based decision processing of appetite stimulation (Grabenhorst \& Rolls, 2011; Jasinska, Ramamoorthy, \& Crew, 2011; Rangel \& Hare, 2010). This finding was in line with previous research where individuals with BN exhibited higher bold activation than HCs in the OFC and right insular cortex, which are implicated in hedonic processing during receipt of food rewards (Brooks et al., 2011; Simon et al., 2016). Meanwhile, previous evidence also showed that patients with BN exhibited decreased activity in reward regions involved in the right anterior insula in response to anticipated receipt of chocolate milkshake (Bohon \& Stice, 2011). A possible explanation for the inconsistency could be that hypofunction of the brain's reward system may reflect an overeating tendency to compensate for the reward system deficit.

We also observed increased DC in the cognitive control system (MFG), and excessive static connections of this region with the MOG and SOG in individuals with sub-BN, as compared to control group. The finding of increased DC in MFG to some extent is similar to those of previous studies, the BN group showed greater neural activation in the MFG within the executive network than did individuals with BED (Stopyra et al., 2019). Compared to HCs, individuals with $B N$ also exhibited greater neural activation in the right dIPFC in response to food images (Brooks et al., 2011). Increased bold activity in the MFG and dIPFC may imply altered cognitive control resources in individuals with BN symptoms. Moreover, our result shows increased MFG-occipital gyrus connectivities. Occipital and parietal lobe activation is involved in the processing of body image (Mele et al., 2020), and DMN regions, such as the medial PFC, posterior cingulate cortex, and temporal cortices were implicated in self-referential processing, which is involved in monitoring physical appearance (Davey, Pujol, \& Harrison, 2016). Previous study also showed that patients with BN had increased FC between the right ventral supramarginal gyrus and all DMN regions (Domakonda et al., 2019), which may reflect their long-term body concerns. Those increased MFG-MOG and MFG-SOG connectivity may reflect a functional change in the connection between self-control and self-referential processing. Individuals with BN mostly have lower explicit self-esteem, including shape/weight-based selfesteem (Cockerham, Stopa, Bell, \& Gregg, 2009), and are in pursue of a perfect body shape. Thus, a possible explanation for the increased MFG-MOG and MFG-SOG connectivity could be that individuals with $\mathrm{BN}$ symptoms might be more motivated to lose weight after losing of control eating, and consequently exhibit heightened cognitive control as an attempt at weight maintenance in the pursuit of the ideal body shape. Therefore, it is possible that additional inhibitory control is warranted to reduce the desire to engage in eating as well as facilitating explicit self-esteem by living up to these standards (Zeigler-Hill \& Terry, 2007). In addition, increased connectivity between the MFG and IFGtriang in individuals with sub-BN was also observed. Analyses further revealed a positive correlation between selfreported BN symptoms and MFG-IFGtriang connectivity. In line between-group findings, this result further indicates that BN symptoms are associated with control motivation-directed goals. Thus, we infer that if BN symptoms were enhanced due to the aberrant activation of reward system, the motivation of weight control may alarm our control system, In other words, the pursuit of the weight control goal might facilitate the processing of cognitive control, however, it's just a bold speculation, as the present evidence so far doesn't answer causation.

Page $11 / 22$ 
Using a seed-based dFC approach, the current study also provided a unique investigation of the dynamics of MFG-whole brain FC associated with the presence of BN symptoms. In support of sFC result, we found significantly decreased MFG-ACC variability in individuals with sub-BN, suggesting increased stability of this connection. The ACC is a prominent part of the executive control system that guides appropriate conflict monitoring (Rogers et al., 2004). In this study, conflict monitoring refers to the regulation between weight control and lose of control eating, while MFG-ACC connectivity indicates that the MFG is involved in the process of regulating these two goals. Accordingly, compared to the control group, more stable MFGACC connectivity possibly implies that the cognitive control system plays an indispensable role in the regulation of monitoring those conflict.

The goal conflict model of eating suggests that chronic restraint dieters have difficulty in regulating their food intake when there is conflict between two incompatible goals, namely, eating enjoyment and weight control (Stroebe et al., 2008). The current findings supported and extended the model by revealing heightened reward processing and cognitive control as well as conflict monitoring brain circuits in the subBN group. Similar to restrained eaters, sub-BN individuals are motivated to pursue their weight-control goal, but they often have uncontrollable desire to binge-eat when tempted by the presence of food (Domakonda et al., 2019). Thus, the conflict experienced between achieving two goals were also salient within this group. Therefore, individuals with sub-BN might face a noticeable struggle between lose of control eating and weight control. In our study this was implicated by the increased activation of the cognitive control and reward systems and by higher connectivity of the cognitive control brain circuit to the self-referential as well as conflict monitoring systems.

In summary, our study revealed aberrant brain activity and connectivity in individuals with sub-BN, implying the importance of brain structures subserving reward value processing, cognitive control, and self-referential processing in these individuals. There were several limitations to this study. Firstly, it had a cross-section rather than longitudinal design; therefore, the developmental changes in brain activity in individuals with sub-BN or patients with $\mathrm{BN}$ could not be clearly investigated. Longitudinal studies are needed to decipher the causal and temporal role of functional abnormalities present in EDs. Secondly, our study used between-group analysis to compare the differences between individuals with BN symptoms and HCs; future studies should use continuous BN symptom variation to find the brain regions of vital importance to clinical BN development. Finally, our study was designed to explore between-group differences of neurobiological mechanisms using resting state fMRI, but little is known about the brain abnormalities of task-based fMRI in individuals with sub-BN; this could exhibit aberrant activation of related brain regions more clearly. Thus, in the future, task-based fMRI and resting-state fMRI should be combined to investigate the overlapping brain regions.

\section{5 | Conclusion}

Results from the present resting-state fMRI study provided evidence of DC, sFC, and dFC changes in subBN. Our findings demonstrate abnormalities in bold activity and connectivity in brain regions involved in reward value processing, cognitive control, and self-referential processing in individuals with sub-BN, 
which supported and extended the goal conflict model of eating in BN. In line with their behaviours, individuals with sub-BN experienced salient conflict between lose of control eating and the goal of weight control, implicated in heightened reward and cognitive control functions. Therefore, individuals with subBN may face a severe struggle between two opposing goals. These results also provide important insights into the pathology of BN.

\section{Declarations}

Ethical approval: All experimental operations were approved by the Southwest University Brain Imaging Center's Institutional Review Board.

Consent to participant: All participants provided written informed consent.

Authors Contributions: L. Luo and Y. J. Luo developed the study concept. All authors contributed to the study design. Data collection was performed by W. Li, Y. Yang and J. J. Wang. Data pre-processing was performed by Xiao, Bian and Leng. L. Luo and X. M. Chen performed the data analysis and interpretation under the supervision of $\mathrm{H}$. Chen. L. Luo and Y. J. Luo drafted the manuscript, and H. Chen provided critical revisions. All authors approved the final version of the manuscript for submission. L. Luo and Y. J. Luo contributed equally to this article and both are responsible for its content.

Funding: This research was funded by National Natural Science Foundation of China (No. 31771237) and the Fundamental Research Funds for the Central Universities (No. SWU1709106).

Competing interests: The authors declare that they have no known competing financial interests or personal relationships that could have appeared to influence the work reported in this paper.

Availability of data and materials lthe data used to support the findings of this study are availability from the corresponding author upon request.

\section{References}

Ashburner J. (2007). A fast diffeomorphic image registration algorithm. Neurolmage, 38(1), 95113. https://doi.org/10.1016/j.neuroimage.2007.07.007

Benninghoven, D., Raykowski, L., Solzbacher, S., Kunzendorf, S., \& Jantschek, G. (2007). Body images of patients with anorexia nervosa, bulimia nervosa and female control subjects: A comparison with male ideals of female attractiveness. Body Image, 4, 51-59. https://doi.org/10.1016/j.bodyim.2006.12.002

Brooks, S. J., O'Daly, O. G., Uher, R., Friederich, H. C., Giampietro, V., Brammer, M., et al.

(2011). Differential neural responses to food images in women with bulimia versus anorexia nervosa. PloS One, 6(7), e22259. https://doi.org/10.1371/journal.pone. 0022259. 
Bohon, C., \& Stice, E. (2011). Reward abnormalities among women with full and subthreshold bulimia nervosa: a functional magnetic resonance imaging study. The International journal of eating disorders, 44(7), 585-595. https://doi.org/10.1002/eat.20869

Burger, K. S., \& Stice, E. (2011). Relation of dietary restraint scores to activation of reward-related brain regions in response to food intake, anticipated intake, and food pictures. Neurolmage, 55(1), 233239. https://doi.org/10.1016/j.neuroimage.2010.12.009

Bernadett, M., \& Szemán-N, A. (2016). Evészavarok prevalenciája policisztás ováriumszindrómás nok körében [Prevalence of eating disorders among women with polycystic ovary syndrome]. Psychiatria Hungarica : A Magyar Pszichiatriai Tarsasag tudomanyos folyoirata, 31(2), 136-145.

Chen, H., Jackson, T., \& Huang, X. (2006). The Negative Physical Self Scale: Initial development and validation in samples of Chinese adolescents and young adults. Body image, 3(4), 401412. https://doi.org/10.1016/j.bodyim.2006.07.005

Crowther, J. H., Armey, M., Luce, K. H., Dalton, G. R., \& Leahey, T. (2008). The point prevalence of bulimic disorders from 1990 to 2004. The International journal of eating disorders, 41(6), 491497. https://doi.org/10.1002/eat.20537

Chen, H., \& Jackson, T. (2008). Prevalence and sociodemographic correlates of eating disorder endorsements among adolescents and young adults from China. European eating disorders review : the journal of the Eating Disorders Association, 16(5), 375-385. https://doi.org/10.1002/erv.837

Coletta, M., Platek, S., Mohamed, F. B., van Steenburgh, J. J., Green, D., \& Lowe, M. R. (2009). Brain activation in restrained and unrestrained eaters: an fMRI study. Journal of abnormal psychology, 118(3), 598-609. https://doi.org/10.1037/a0016201

Cockerham, E., Stopa, L., Bell, L., \& Gregg, A. (2009). Implicit self-esteem in bulimia nervosa. Journal of behavior therapy and experimental psychiatry, 40(2), 265-273.

https://doi.org/10.1016/j.jbtep.2008.12.003

Calhoun, V. D., Miller, R., Pearlson, G., \& Adalı, T. (2014). The chronnectome: time-varying connectivity networks as the next frontier in fMRI data discovery. Neuron, 84(2), 262-

274. https://doi.org/10.1016/j.neuron.2014.10.015

Cyr, M., Kopala-Sibley, D. C., Lee, S., Chen, C., Stefan, M., Fontaine, M., et al. (2017). Reduced inferior and orbital frontal thickness in adolescent bulimia nervosa persists over two-year follow-up. Journal of the American Academy of Child \& Adolescent Psychiatry, 56(10), 866874. https://doi.org/10.1016/j.jaac.2017.08.008.

Davey, C. G., Pujol, J., \& Harrison, B. J. (2016). Mapping the self in the brain's default mode network. Neurolmage, 132, 390-397. https://doi.org/10.1016/j.neuroimage.2016.02.022 
Domakonda, M. J., He, X., Lee, S., Cyr, M., \& Marsh, R. (2019). Increased Functional Connectivity Between Ventral Attention and Default Mode Networks in Adolescents With Bulimia Nervosa. Journal of the American Academy of Child and Adolescent Psychiatry, 58(2), 232-241.

https://doi.org/10.1016/j.jaac.2018.09.433

Friston, K. J., Williams, S., Howard, R., Frackowiak, R. S., \& Turner, R. (1996). Movement-related effects in $\mathrm{fMRI}$ time-series. Magnetic resonance in medicine, 35(3), 346-

355. https://doi.org/10.1002/mrm.1910350312

Frank, G. K., Wagner, A., Achenbach, S., McConaha, C., Skovira, K., Aizenstein, H., Carter, C. S., \& Kaye, W. H. (2006). Altered brain activity in women recovered from bulimic-type eating disorders after a glucose challenge: a pilot study. The International journal of eating disorders, 39(1), 76-

79. https://doi.org/10.1002/eat.20210

Fox, M. D., \& Raichle, M. E. (2007). Spontaneous fluctuations in brain activity observed with functional magnetic resonance imaging. Nature reviews. Neuroscience, 8(9), 700-

711. https://doi.org/10.1038/nrn2201

Grabenhorst, F., \& Rolls, E. T. (2011). Value, pleasure and choice in the ventral prefrontal cortex. Trends in cognitive sciences, 15(2), 56-67. https://doi.org/10.1016/j.tics.2010.12.004

Heatherton, T. F., \& Wagner, D. D. (2011). Cognitive neuroscience of self-regulation failure. Trends in cognitive sciences, 15(3), 132-139. https://doi.org/10.1016/j.tics.2010.12.005

Hallquist, M. N., Hwang, K., \& Luna, B. (2013). The nuisance of nuisance regression: spectral misspecification in a common approach to resting-state fMRI preprocessing reintroduces noise and obscures functional connectivity. Neurolmage, 82, 208225. https://doi.org/10.1016/j.neuroimage.2013.05.116

Hutchison, R. M., Womelsdorf, T., Allen, E. A., Bandettini, P. A., Calhoun, V. D., Corbetta, M., Della Penna, S., Duyn, J. H., Glover, G. H., Gonzalez-Castillo, J., Handwerker, D. A., Keilholz, S., Kiviniemi, V., Leopold, D. A., de Pasquale, F., Sporns, O., Walter, M., \& Chang, C. (2013). Dynamic functional connectivity: promise, issues, and interpretations. Neurolmage, 80, 360-378. https://doi.org/10.1016/j.neuroimage.2013.05.079

Joos, A. A., Saum, B., Zeeck, A., Perlov, E., Glauche, V., Hartmann, A., Freyer, T., Sandholz, A., Unterbrink, T., van Elst, L. T., \& Tüscher, O. (2011). Frontocingular dysfunction in bulimia nervosa when confronted with disease-specific stimuli. European eating disorders review : the journal of the Eating Disorders Association, 19(5), 447-453. https://doi.org/10.1002/erv.1150

Jasinska, A. J., Ramamoorthy, A., \& Crew, C. M. (2011). Toward a neurobiological model of cue-induced self-control in decision making: relevance to addiction and obesity. The Journal of neuroscience : the official journal of the Society for Neuroscience, 31(45), 1613916141. https://doi.org/10.1523/JNEUROSCI.4477-11.2011 
Kong, F., Zhang, Y., \& Chen, H. (2013). The construct validity of the Restraint Scale among mainland Chinese women. Eating behaviors, 14(3), 356-360. https://doi.org/10.1016/j.eatbeh.2013.06.009

Lee, S., Kim, R. K., Ku, J., Lee, J. H., Namkoong, K., \& Jung, Y. C. (2014). Resting-state synchrony between anterior cingulate cortex and pre- cuneus relates to body shape concern in anorexia nervosa and bulimia nervosa. Psychiatry Research: Neuroimaging, 221, 4348. https://doi.org/10.1016/j.pscychresns.2013.11.004

Liao, W., Wu, G. R., Xu, Q., Ji, G. J., Zhang, Z., Zang, Y. F., \& Lu, G. (2014). DynamicBC: a MATLAB toolbox for dynamic brain connectome analysis. Brain connectivity, 4(10), 780-

790. https://doi.org/10.1089/brain.2014.0253

Leonardi, N., \& Van De Ville, D. (2015). On spurious and real fluctuations of dynamic functional connectivity during rest. Neurolmage, 104, 430-436. https://doi.org/10.1016/j.neuroimage.2014.09.007

Lyu, Z., Zheng, P., \& Jackson, T. (2016). Attention Disengagement Difficulties among Average Weight Women Who Binge Eat. European eating disorders review : the journal of the Eating Disorders Association, 24(4), 286-293. https://doi.org/10.1002/erv.2438

Li, R., Liao, W., Yu, Y., Chen, H., Guo, X., Tang, Y. L., \& Chen, H. (2018). Differential patterns of dynamic functional connectivity variability of striato-cortical circuitry in children with benign epilepsy with centrotemporal spikes. Human brain mapping, 39(3), 1207-1217. https://doi.org/10.1002/hbm.23910

Li-ning DUAN, Yi-feng XU, Min LI (2020). Acupoint catgut embedding combined with auricular plaster

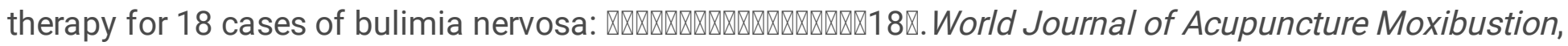
30(4), 304-306.https://doi.org/10.1016/j.wjam.2020.10.002

Mennes, M., Kelly, C., Zuo, X. N., Di Martino, A., Biswal, B. B., Castellanos, F. X., \&

Westwater, M. L., Seidlitz, J., Diederen, K. M. J., Fischer, S., \& Thompson, J. C. (2018). Associations between cortical thickness, structural connectivity and severity of dimensional bulimia nervosa symptomatology. Psychiatry Research Neuroimaging,30(271), 118-125. https://doi.org/10.1016/j.pscychresns.2017.11.006.

Mendelson BK, White DR, MJ (1996). Manual for the Body-Esteem Scale for Adolescents and Adults[J]. Journal of Applied Developental Psychology, 17: 321-346

Meijboom, A., Jansen, A., Kampman, M., \& Schouten, E. (1999). An experimental test of the relationship between self-esteem and concern about body shape and weight in restrained eaters. The International journal of eating disorders, 25(3), 327-334. https://doi.org/10.1002/(sici)1098$108 \times(199904) 25: 3<327:$ :aid-eat11>3.0.co;2-5

Milham, M. P. (2010). Inter-individual differences in resting-state functional connectivity predict taskinduced BOLD activity. Neurolmage, 50(4), 1690- 
Mele, G., Alfano, V., Cotugno, A., \& Longarzo, M. (2020). A broad-spectrum review on multimodal neuroimaging in bulimia nervosa and binge eating disorder. Appetite, 151, 104712. https://doi.org/10.1016/j.appet.2020.104712

Neveu, R., Neveu, D., Carrier, E., Gay, A., Nicolas, A., \& Coricelli, G. (2018). Goal Directed and Self-Control Systems in Bulimia Nervosa: An fMRI Study. EBioMedicine, 34, 214222. https://doi.org/10.1016/j.ebiom.2018.07.012

Mak, L. E., Minuzzi, L., MacQueen, G., Hall, G., Kennedy, S. H., \& Milev, R. (2017). The Default Mode Network in Healthy Individuals: A Systematic Review and Meta-Analysis. Brain connectivity, 7(1), 25-33. https://doi.org/10.1089/brain.2016.0438

Power, J. D., Barnes, K. A., Snyder, A. Z., Schlaggar, B. L., \& Petersen, S. E. (2012). Spurious but systematic correlations in functional connectivity MRI networks arise from subject motion. Neurolmage, 59(3), 21422154. https://doi.org/10.1016/j.neuroimage.2011.10.018

Pan, W., Liu, C., Yang, Q., Gu, Y., Yin, S., \& Chen, A. (2016). The neural basis of trait self-esteem revealed by the amplitude of low-frequency fluctuations and resting state functional connectivity. Social cognitive and affective neuroscience, 11(3), 367-376. https://doi.org/10.1093/scan/nsv119

Pryor, Tamara \& Frank, Guido \& Shott, Megan \& Riederer, J. (2016). Altered structural and effective connectivity in anorexia and bulimia nervosa in circuits that regulate energy and reward homeostasis. Translational Psychiatry. 6. 10.1038/tp.2016.199.

Preti, M. G., Bolton, T. A., \& Van De Ville, D. (2017). The dynamic functional connectome: State-of-the-art and perspectives. Neurolmage, 160, 41-54. https://doi.org/10.1016/j.neuroimage.2016.12.061

Rogers, R. D., Ramnani, N., Mackay, C., Wilson, J. L., Jezzard, P., Carter, C. S., \& Smith, S. M. (2004). Distinct portions of anterior cingulate cortex and medial prefrontal cortex are activated by reward processing in separable phases of decision-making cognition. Biological psychiatry, 55(6), 594602. https://doi.org/10.1016/j.biopsych.2003.11.012

Rangel, A., \& Hare, T. (2010). Neural computations associated with goal-directed choice. Current opinion in neurobiology, 20(2), 262-270. https://doi.org/10.1016/j.conb.2010.03.001

Stice, E., Telch, C. F., \& Rizvi, S. L. (2000). Development and validation of the Eating Disorder Diagnostic Scale: a brief self-report measure of anorexia, bulimia, and binge-eating disorder. Psychological assessment, 12(2), 123-131. https://doi.org/10.1037//1040-3590.12.2.123

Stice, E., Fisher, M., \& Martinez, E. (2004). Eating disorder diagnostic scale: additional evidence of reliability and validity. Psychological assessment, 16(1), 60-71. https://doi.org/10.1037/1040-3590.16.1.60 
Stice, E., \& Desjardins, C. D. (2018). Interactions between risk factors in the prediction of onset of eating disorders: Exploratory hypothesis generating analyses. Behaviour research and therapy, 105, 52-62. https://doi.org/10.1016/j.brat.2018.03.005

Stroebe, W., Mensink, W., Aarts, H., Schut, H., \& Kruglanski, A. W. (2008). Why dieters fail: Testing the goalconflict model of eating. Journal of Experimental Social Psychology, 44, 26-36.

Seitz, J., Hueck, M., Dahmen, B., Schulte-Rüther, M., Legenbauer, T., Herpertz-Dahlmann, B., \& Konrad, K. (2016). Attention Network Dysfunction in Bulimia Nervosa - An fMRI Study. PloS one, 11(9), e0161329. https://doi.org/10.1371/journal.pone.0161329

Simon, J. J., Skunde, M., Walther, S., Bendszus, M., Herzog, W., \& Friederich, H. C. (2016). Neural signature of food reward processing in bulimic-type eating disorders. Social Cognitive and Affective Neuroscience, 11(9), 1393-1401. https://doi.org/10. 1093/scan/nsw049.

Spalatro, A. V., Amianto, F., Huang, Z., D'Agata, F., Bergui, M., Abbate Daga, G., Fassino, S., \& Northoff, G. (2019). Neuronal variability of Resting State activity in Eating Disorders: increase and decoupling in Ventral Attention Network and relation with clinical symptoms. European psychiatry : the journal of the Association of European Psychiatrists, 55, 10-17. https://doi.org/10.1016/j.eurpsy.2018.08.005]

Stopyra, M. A., Simon, J. J., Skunde, M., Walther, S., Bendszus, M., Herzog, W., \& Friederich, H. C. (2019). Altered functional connectivity in binge eating disorder and bulimia nervosa: A resting-state fMRI study.Brain and behavior, 9(2), e01207. https://doi.org/10.1002/brb3.1207

Vatansever, D., Manktelow, A., Sahakian, B. J., Menon, D. K., \& Stamatakis, E. A. (2018). Default Mode Network Engagement Beyond Self-Referential Internal Mentation. Brain connectivity, 8(4), 245-253. https://doi.org/10.1089/brain.2017.0489

Worsley KJ, Friston KJ. Analysis of fMRI time-series revisited-again. Neuroimage. 1995 Sep;2(3):173-81. doi: 10.1006/nimg.1995.1023. PMID: 9343600.

Wood, S. M., Schembre, S. M., He, Q., Engelmann, J. M., Ames, S. L., \& Bechara, A. (2016). Emotional eating and routine restraint scores are associated with activity in brain regions involved in urge and self-control. Physiology \& behavior, 165, 405-412. https://doi.org/10.1016/j.physbeh.2016.08.024

Wang, X., Jiao, D., Zhang, X., \& Lin, X. (2017). Altered degree centrality in childhood absence epilepsy: A resting-state fMRI study. Journal of the neurological sciences, 373, 274279. https://doi.org/10.1016/j.jns.2016.12.054

Wang, L., Bi, K., An, J., Li, M., Li, K., Kong, Q. M., Li, X. N., Lu, Q., \& Si, T. M. (2019). Abnormal structural brain network and hemisphere-specific changes in bulimia nervosa. Translational psychiatry, 9(1), 206. https://doi.org/10.1038/s41398-019-0543-1 
Wallace, G. L., Richard, E., Peng, C. S., Knodt, A. R., \& Hariri, A. R. (2019). Subclinical eating disorder traits are correlated with cortical thickness in regions associated with food reward and perception. Brain Imaging and Behavior, 8. https://doi.org/10.1007/s11682-018-0007-x.

Wade T. D. (2019). Recent Research on Bulimia Nervosa. The Psychiatric clinics of North America, 42(1), 21-32.https://doi.org/10.1016/j.psc.2018.10.002

Yan, C. G., Wang, X. D., Zuo, X. N., \& Zang, Y. F. (2016). DPABI: Data Processing \& Analysis for (RestingState) Brain Imaging. Neuroinformatics, 14(3), 339-351. https://doi.org/10.1007/s12021-016-9299-4

Zeigler-Hill, V., Terry, C. (2007). Perfectionism and Explicit Self-Esteem: The Moderating Role of Implicit Self-Esteem. Self and Identity, 6(41308), 137-153.Available at: https://aquila.usm.edu/fac_pubs/2039

Zhou, Y., Wang, Y., Rao, L. L., Liang, Z. Y., Chen, X. P., Zheng, D., Tan, C., Tian, Z. Q., Wang, C. H., Bai, Y. Q., Chen, S. G., \& Li, S. (2014). Disrutpted resting-state functional architecture of the brain after 45-day simulated microgravity. Frontiers in behavioral neuroscience, 8 , 200. https://doi.org/10.3389/fnbeh.2014.00200

Zhang, X., Liu, Y., Gu, Y., Wang, S., \& Chen, H. (2020). Red for "Stop": "Traffic-Light" Nutrition Labels Decrease Unhealthy Food Choices by Increasing Activity and Connectivity in the Frontal Lobe. Nutrients, 12(1), 128. https://doi.org/10.3390/nu12010128

\section{Figures}




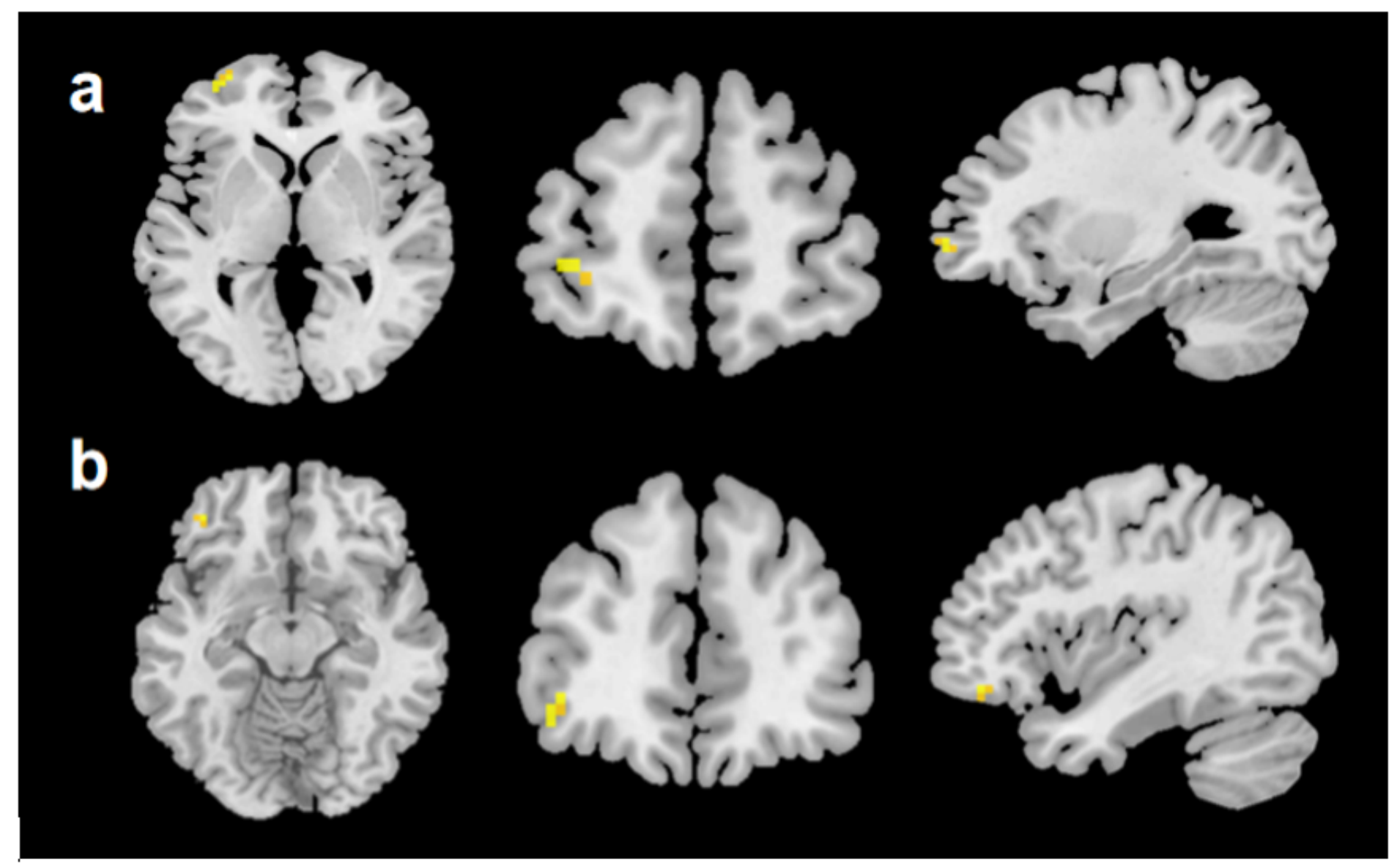

Figure 1

Views of significant changes in DC between two groups. Sub-BN showed significantly increased DC in (a) the MFG and (b) the OFG (marked in yellow). Abbreviations: sub-BN = subthreshold bulimia nervosa, $\mathrm{HC}$ =control group, $\mathrm{DC}=$ degree centrality, $\mathrm{MFG}=$ middle frontal gyrus, $\mathrm{OFG}=$ orbital frontal gyrus. 
(a)
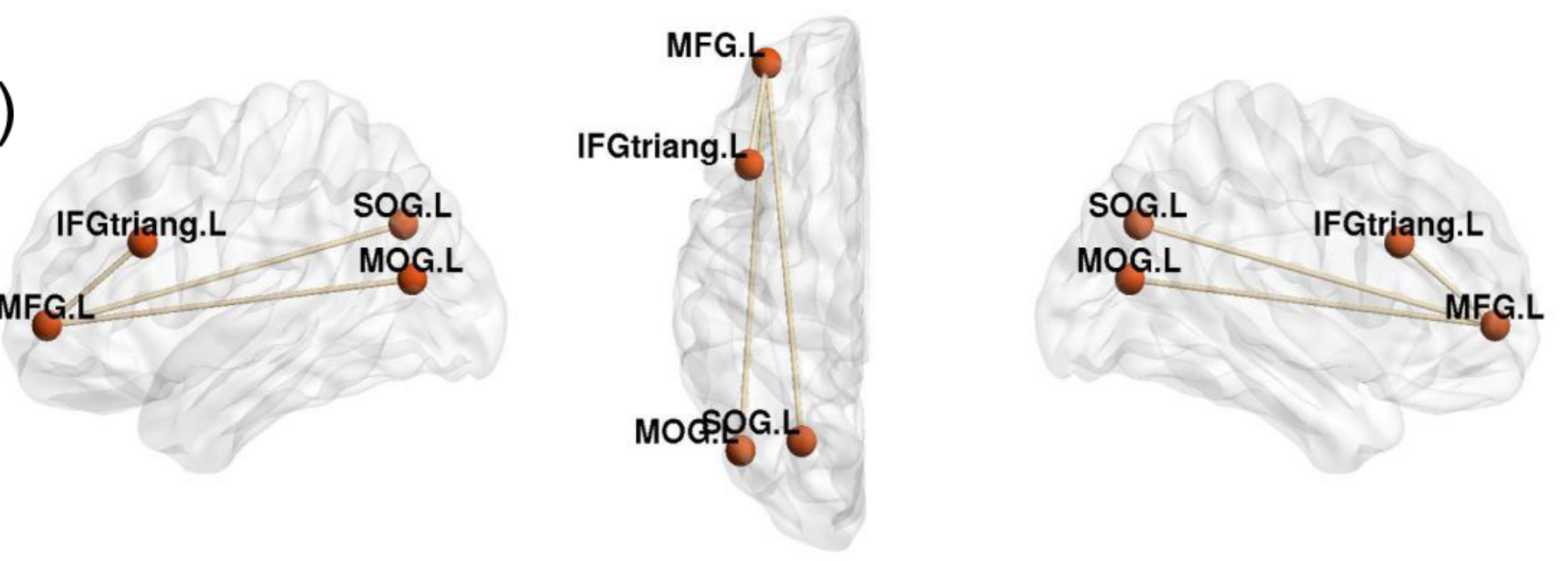

(b)

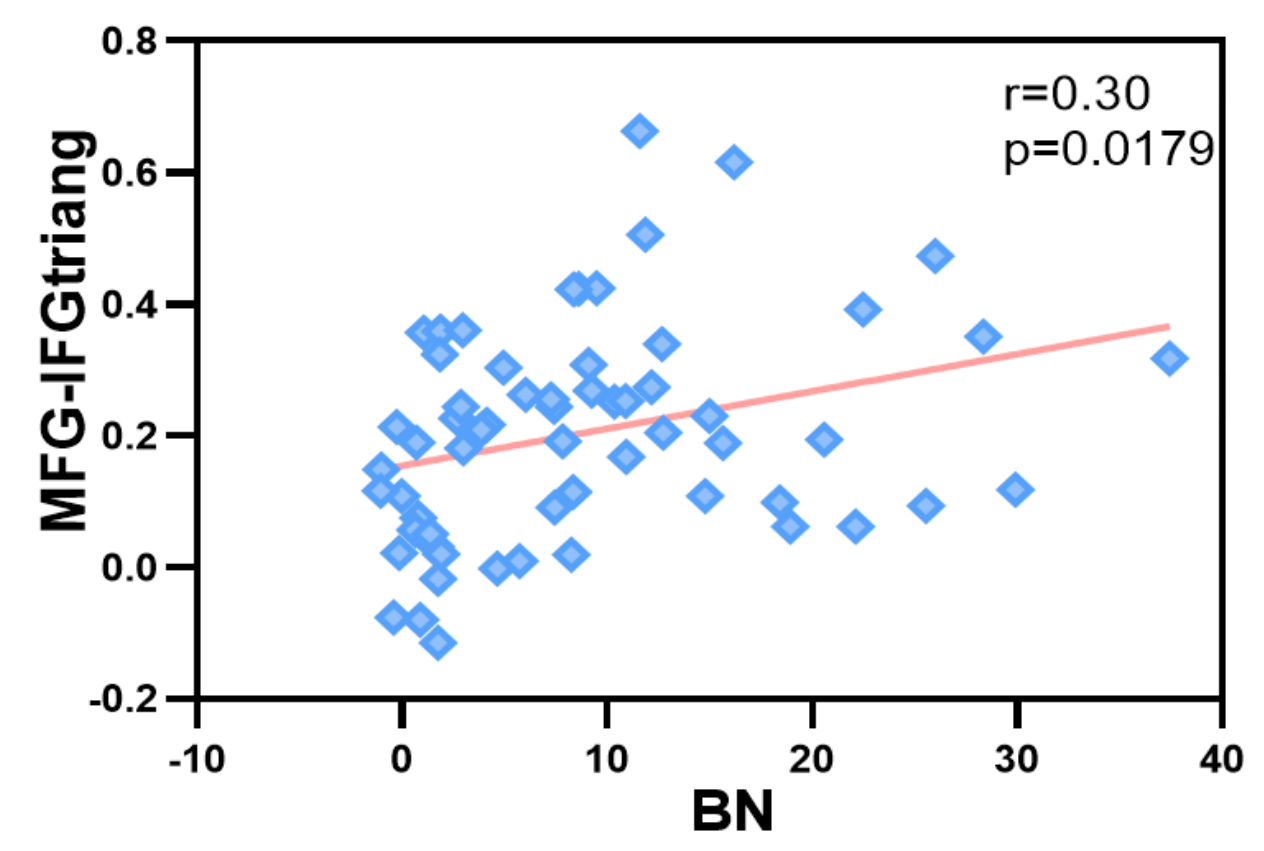

Figure 2

(a) Between-group differences of three MFG-based sFC (Gaussian random field correction $p<0.05$ at cluster level and $p<0.005$ at voxel level, two-tailed) (b) Positive correlations between the MFG-IFGtriang and self-reported $B N$ symptoms in the sub-BN group $(r=0.30, p=0.018)$. Abbreviations: Sub-BN = subthreshold bulimia nervosa, $\mathrm{HC}=$ control group, $\mathrm{L}=$ left, $\mathrm{R}=$ right, MFG = middle frontal gyrus, IFGtriang $=$ triangular part of the inferior frontal gyrus, $\mathrm{SOG}=$ superior occipital gyrus, MOG = middle occipital gyrus . 
(b)

$\mathbf{R}$
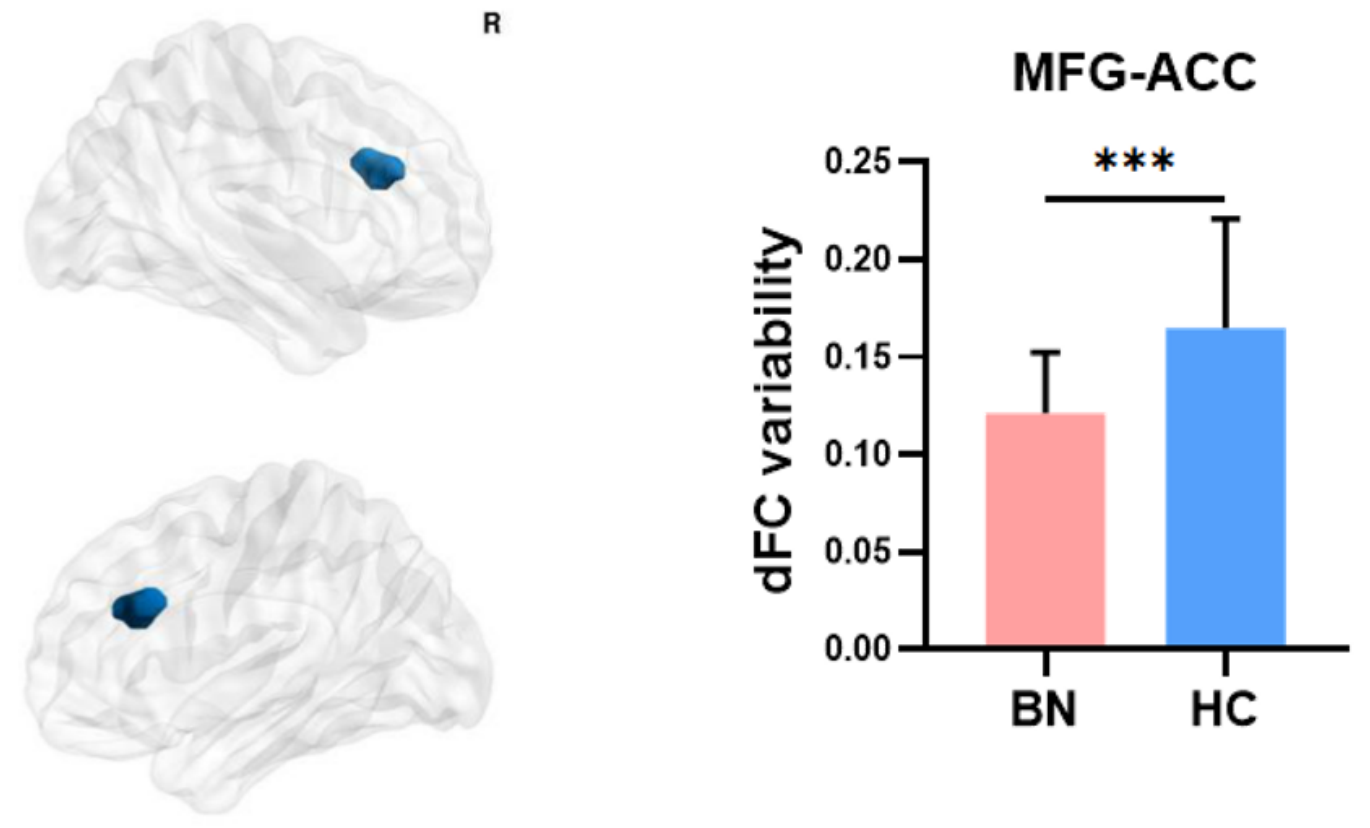

Figure 3

(a) Between-group difference in MFG-ACC dFC variability (Gaussian random field correction $p<0.05$ at cluster level and $p<0.005$ at voxel level, two-tailed) (b) Bar chart showing the significant group difference in MFG-ACC dFC. Abbreviations: $\mathrm{R}=$ right, MFG = middle frontal gyrus, $\mathrm{ACC}=$ anterior cingulate cortex, dFC $=$ dynamic functional connectivity.

\section{Supplementary Files}

This is a list of supplementary files associated with this preprint. Click to download.

- BIBChecklist.docx 